\title{
Many-spinon states and the secret significance of Young tableaux
}

\author{
Martin Greiter and Dirk Schuricht \\ Institut für Theorie der Kondensierten Materie, Universität Karlsruhe, \\ Postfach 6980, 76128 Karlsruhe, Germany
}

\begin{abstract}
We establish a one-to-one correspondence between the Young tableaux classifying the total spin representations of $N$ spins and the exact eigenstates of the the Haldane-Shastry model for a chain with $N$ sites classified by the total spins and the fractionally spaced single-particle momenta of the spinons.
\end{abstract}

PACS numbers: 75.10.Pq, 02.30.Ik, 05.30.Pr, 02.20.-a

Integrable models [1, 2] have always played a special role in our understanding of interacting many-particle systems in one dimension. While it is clearly impossible to realize an integrable model experimentally, it is clear that many concepts and mechanisms discovered through the study of integrable systems are directly or indirectly relevant to a substantial body of experimental data. An infinite set of conserved quantities renders many integrable models amenable to exact solution through one or several variations of the Bethe ansatz method, and hence allows us to get a grasp on the non-trivial physical concepts involved. The most prominent among these concepts is probably the fractional quantization of spin in antiferromagnetic spin $1 / 2$ chains. Faddeev and Takhtajan [3] discovered in 1981 that the elementary excitations (now called spinons) of the spin $1 / 2$ Heisenberg chain solved by Bethe [4] in 1931 carry spin 1/2 while the Hilbert space for the spin chain is spanned by spin flips, which carry spin 1 . The fractional quantization of spin in spin chains is conceptually very similar to the fractional quantization of charge in quantized Hall liquids [5, 6]. In the case of the Haldane-Shastry model (HSM) [7, 8, 9, 10, 11], which we will elaborate on below, the analogy even extends to the explicit wave functions for the ground states and the spinon or quasihole excitations of the spin chain and the Hall liquid, respectively.

The discovery of the spinon through the Bethe ansatz (BA) solution illustrates both the importance of integrable models and BA techniques as well as the practical limitations, as it took 50 years to read off as elementary a property as the spin of an excitation in a known and established exact solution to the problem. The BA is not a very practical method to calculate observable quantities, both because the number of integrable models is limited and because it is often exceedingly difficult to extract physical quantities like correlation or response functions from the exact solutions. One of the reasons underlying these practical limitations may be that the BA solutions are given as distributions of pseudomomenta, in which spinon excitations appear as defects. Spinons hence play the role of defects or solitons in the solutions of the BA equations, just as they and the quasiparticles in quantized Hall liquids are often viewed as solitons or collective excitations in states constructed of spin flips or electrons, respectively. In many regards, however, spinons can and should be interpreted as particles, which requires solutions labelled in terms of spinon quantum numbers. Such solutions would, in principle, allow for the development of perturbative methods in the spinon fields directly.

With regard to this perspective, there is encouragement and there are problems. The encouraging news is that the HSM provides us with an ideal starting point for any such perturbative expansion, since the spinons in this model are free [12, 13, 14] in the sense that they interact only through their half-Fermi statistics. The half-Fermi statistics yields non-trivial state counting rules [15, 16, 17] and fractional momentum spacings [18]. It is still far from simple to construct the spinon Hilbert space, as it cannot be decomposed into a product space of single-particle states, known as Fock space in the familiar cases of fermions or bosons. In this Letter, we propose what we believe to be the simplest construction.

The main problem with the development of perturbative methods in terms of spinon fields is that the matrix elements of local spin operators $\boldsymbol{S}_{\alpha}$ on sites $\alpha$ of the chain between states with different numbers of free spinons, i.e., eigenstates of the HSM, are required. At present, only very few of these matrix elements are known, and the exact expressions for these elements for finite chains appear rather complicated [19, 20, 21]. These expressions, however, greatly simplify in the thermodynamic limit, and there is hope that a method to obtain them directly in this limit can be developed.

Let us now turn to the Hilbert space representation for spinons in an $\mathrm{SU}(2)$ or, in general, $\mathrm{SU}(n)$ spin chain. The non-trivial feature is that the single-spinon quantum numbers depend on the occupations of all the other single-spinon states. These quantum numbers are the spins and the single-particle momenta of the spinons. It would probably be exceedingly difficult to obtain them if the Haldane-Shastry model would not provide us with a framework. Since the spinons in this model are free, the problem of finding the equivalent of a Fock space representation for spinons reduces to finding a convenient representation of the eigenstates of this model in terms of their spinon content. In other words, we set out to find a general set of rules to determine the allowed sequences of single-spinon momenta $p_{1}, \ldots, p_{L}$ as well as the allowed representations for the total spin of the states such that the eigenstates of the HSM have momenta and energies

$$
p=p_{0}+\sum_{i=1}^{L} p_{i}, \quad E=E_{0}+\sum_{i=1}^{L} \epsilon\left(p_{i}\right)
$$


where $p_{0}$ and $E_{0}$ denote the ground state momentum and energy, respectively, and $\epsilon(p)$ a single-spinon dispersion irrelevant to the general purpose. We begin with a brief review of the most relevant features of the model.

The HSM is most conveniently formulated by embedding the one-dimensional chain with periodic boundary conditions into the complex plane by mapping it onto the unit circle with the spins located at complex positions $\eta_{\alpha}=\exp \left(i \frac{2 \pi}{N} \alpha\right)$, where $N$ denotes the number of sites and $\alpha=1, \ldots, N$. The Hamiltonian is given by

$$
H_{\mathrm{HS}}=\frac{2 \pi^{2}}{N^{2}} \sum_{\alpha<\beta}^{N} \frac{P_{\alpha \beta}}{\left|\eta_{\alpha}-\eta_{\beta}\right|^{2}},
$$

where $P_{\alpha \beta}$ permutes the spins on sites $\alpha$ and $\beta$. For the $\mathrm{SU}(2)$ model, (2) takes the more familiar form if we substitute $P_{\alpha \beta}=2 \boldsymbol{S}_{\alpha} \cdot \boldsymbol{S}_{\beta}+\frac{1}{2}$. The model is integrable 22. and possesses a Yangian symmetry algebra generated by the total spin $\boldsymbol{S}$ and the rapidity operator $\boldsymbol{\Lambda}$, which both commute with the Hamiltonian but do not commute mutually [11].

The ground state ( $N=n M, M$ integer) for the $\mathrm{SU}(2)$ model $(n=2)$ is given by

$$
\left|\Psi_{0}\right\rangle=P_{\mathrm{G}}\left|\Psi_{\mathrm{SD}}^{N}\right\rangle, \quad\left|\Psi_{\mathrm{SD}}^{N}\right\rangle \equiv \prod_{q \in \mathcal{I}} c_{q \uparrow}^{\dagger} c_{q \downarrow}^{\dagger}|0\rangle
$$

where the Gutzwiller projector $P_{\mathrm{G}}$ eliminates configurations with more than one particle on any site and the interval $\mathcal{I}$ contains $M$ adjacent momenta. For $\mathrm{SU}(n)$, each momenta in $\mathcal{I}$ has to be occupied by $n$ particles with different spins [23, 24]. As the $N$-particle Slater-determinant state $\left|\Psi_{\mathrm{SD}}^{N}\right\rangle$ is a spin singlet by construction and $P_{\mathrm{G}}$ commutes with $\mathrm{SU}(n)$ rotations, $\left|\Psi_{0}\right\rangle$ is an $\mathrm{SU}(n)$ singlet as well.

A non-orthogonal but complete basis for spin-polarized two-spinon eigenstates with total momentum $p=-k_{1}-k_{2}$ is given by

$$
\left|\Psi_{p_{1} \uparrow, p_{2} \uparrow}\right\rangle=P_{\mathrm{G}} c_{k_{1} \downarrow} c_{k_{2} \downarrow}\left|\Psi_{\mathrm{SD}}^{N+2}\right\rangle, \quad k_{1}>k_{2} .
$$

These states are not eigenstates, but as $H_{\mathrm{HS}}$ scatters $k_{1}$ and $k_{2}$ in only one direction (increasing $k_{1}-k_{2}$ ), there is a one-to-one correspondence between these basis states and the exact eigenstates constructed by superposition. The total energy of the eigenstates takes the form (1) if and only if the single-spinon momenta are shifted with respect to $k_{1,2}$ [14, 25]:

$$
p_{1,2}=-k_{1,2} \pm \frac{1}{2 n} \frac{2 \pi}{N}, \quad p_{1}<p_{2} .
$$

The shift can be interpreted as manifestation of the fractional statistics of the spinons [15, 18]. For $\mathrm{SU}(2)$, an energetically degenerate two-spinon singlet state $\Lambda^{z} S^{-}\left|\Psi_{p_{1} \uparrow, p_{2} \uparrow}\right\rangle$ with the same single-spinon momenta exists only for $p_{2}-p_{1}>\frac{1}{2} \frac{2 \pi}{N}$, as (15) is annihilated by $\Lambda^{z} S^{-}$ for $p_{2}-p_{1}=\frac{1}{2} \frac{2 \pi}{N}$ [11]. These features illustrate that the rules specifying the allowed single-spinon momenta and spin representations are non-trivial.

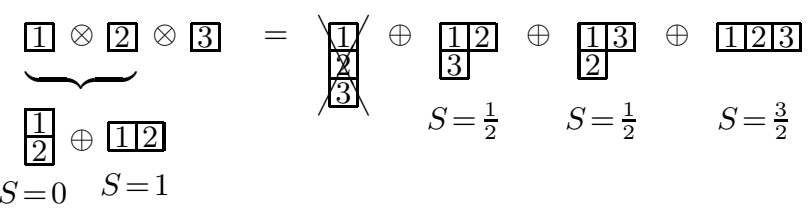

FIG. 1: Total spin representations of three $S=\frac{1}{2}$ spins with Young tableaux. For $\mathrm{SU}(n)$ with $n>2$, the tableaux with three boxes on top of each other exists as well.

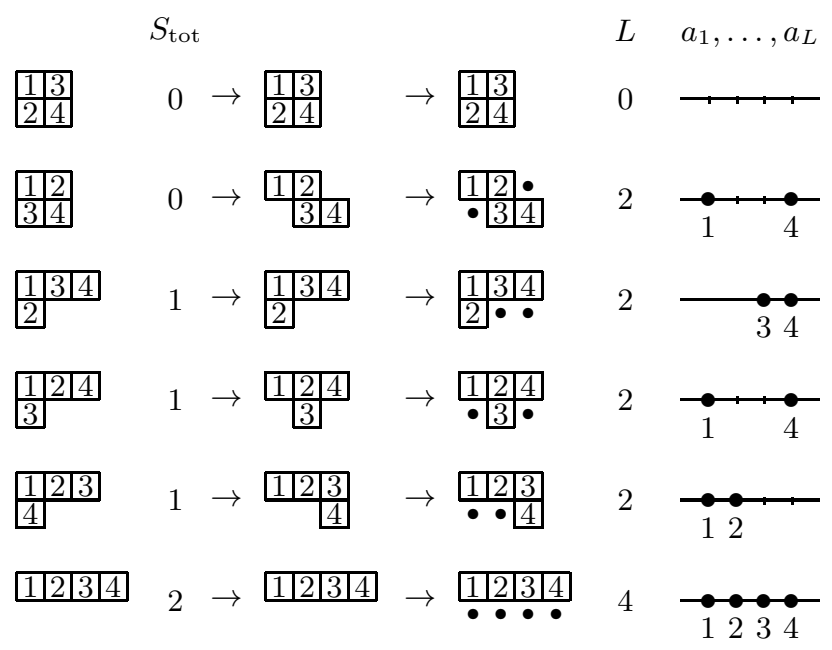

FIG. 2: Young tableau decomposition and the corresponding spinon states for an $S=\frac{1}{2}$ spin chain with $N=4$ sites. The dots represent the spinons. For $\mathrm{SU}(2)$, the spinon momentum numbers $a_{i}$ are given by the numbers in the boxes of the same column. Note that $\sum\left(2 S_{\text {tot }}+1\right)=2^{N}$.

We now proceed by stating these rules without further motivating or even deriving them. To begin with, the Hilbert space of a system of $N$ identical $\mathrm{SU}(n)$ spins can be decomposed into representations of the total spin, which commutes with (2) and hence can be used to classify the eigenstates. This decomposition can be obtained using Young tableaux [26], as illustrated for three $S=\frac{1}{2}$ spins in Fig. 1. The general rule is as follows. For each of the $N$ spins, draw a box numbered consecutively from left to right. The representations of $\mathrm{SU}(n)$ are constructed by putting the boxes together such that the numbers assigned to them increase in each row from left to right and in each column from top to bottom. Each tableau indicates symmetrization over all boxes in the same row, and antisymmetrization over all boxes in the same column. This implies that we cannot have more than $n$ boxes on top of each other for $\mathrm{SU}(n)$ spins. For $\mathrm{SU}(2)$, each tableau corresponds to a spin $S=\frac{1}{2}\left(\lambda_{1}-\lambda_{2}\right)$ representation, with $\lambda_{i}$ the number of boxes in the $i$ th row, and stands for a multiplet $S^{z}=-S, \ldots, S$.

The main result presented in this Letter is that there is a one-to-one correspondence between these Young tableaux and the non-interacting many-spinon states, i.e., the eigenstates of the HSM. The general principle is illus- 
trated for an $\mathrm{SU}(2)$ chain with four sites in Fig. 2, and for a few representations of an $\mathrm{SU}(3)$ chain with six sites in Fig. 3. The rule is that in each Young tableau, we shift boxes to the right such that each box is below or in the column to the right of the box with the preceding number. Each missing box in the resulting, extended tableaux represents a spinon. The extended tableaux provide us with the total spin of each multiplet, which is given by the representation specified by the original Young tableau, as well as the number $L$ of spinons present and the individual single-spinon momenta $p_{1}, \ldots, p_{L}$. The latter yield the kinetic energy of the many-spinon state, i.e., the energy of the corresponding eigenstate of the HSM. We will elaborate on this now.

To begin with, we introduce a spinon momentum number (SMN) $a_{i}$ for each spinon. For an $\mathrm{SU}(2)$ chain, it is simply given by the number in the box in the same column (see Fig. 2). For a general $\mathrm{SU}(n)$ chain, the SMNs for the spinons in each column are given by a sequence of numbers (half-integer for $n$ odd, integers for $n$ even) with integer spacings such that the arithmetic mean equals the arithmetic mean of the numbers in the boxes of the column. To give an example, consider the extended tableau in the second line in Fig. 3. In the first column, there is only one box, and the arithmetic mean of the numbers in the boxes is trivially 1 . The SMNs for the two spinons are hence $\frac{1}{2}$ and $\frac{3}{2}$, as these are integer spaced and have likewise arithmetic mean 1 . In the last column, there is only one spinon. The SMN is given by the arithmetic mean of the two numbers in the boxes, i.e., $(5+6) / 2=\frac{11}{2}$. The individual spinon momenta corresponding to SMNs $a_{i}$ are simply given by

$$
p_{i}=\frac{2 \pi}{N} \frac{a_{i}-\frac{1}{2}}{n},
$$

which implies $\delta \leq p_{i} \leq \frac{2 \pi}{n}-\delta$ with $\delta=\frac{2 \pi}{N}\left(\frac{3}{2 n}-\frac{1}{2}\right) \rightarrow 0$ for $N \rightarrow \infty$. For $\mathrm{SU}(2)$, the total momentum and $\mathrm{HS}$ energies of the many-spinon states are given by (11) with

$$
p_{0}=-\frac{\pi}{2} N, \quad E_{0}=-\frac{\pi^{2}}{4 N},
$$

and the single-spinon dispersion

$$
\epsilon(p)=\frac{1}{2} p(\pi-p)+\frac{\pi^{2}}{8 N^{2}},
$$

where we use a convention according to which the "vacuum" state $|\downarrow \downarrow \ldots \downarrow\rangle$ has momentum $p=0$ (and the empty state $|0\rangle$ has $p=\pi$ ). The corresponding formulas for $\mathrm{SU}(n)$ are

$$
p_{0}=-\frac{(n-1) \pi}{n} N, \quad E_{0}=-\frac{\pi^{2}}{12}\left(\frac{n-2}{n} N+\frac{2 n-1}{N}\right),
$$

and

$$
\epsilon(p)=\frac{n}{4} p\left(\frac{2 \pi}{n}-p\right)+\frac{n^{2}-1}{12 n} \frac{\pi^{2}}{N^{2}} .
$$

The simple formalism we just presented provides the complete spectrum of the HSM.

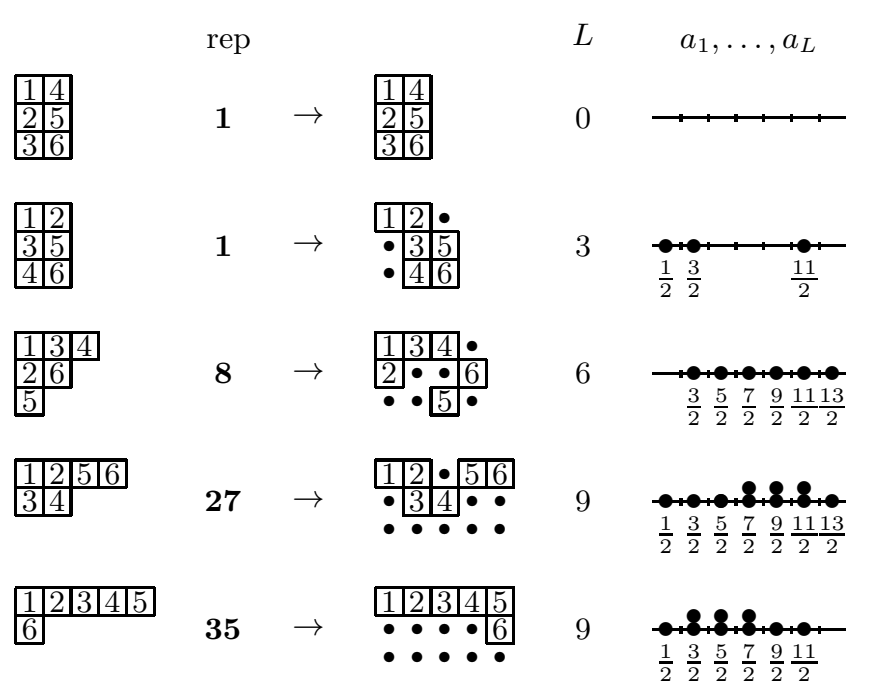

FIG. 3: Examples of eigenstates of the SU(3) HSM with $N=6$ sites in terms of colorons.

These results were obtained heuristically, initially being hardly more than an educated guess at what the equivalent of Fock space for spinons might be. It is easy to see that the momentum spacings for spin polarized spinons predicted by this formalism reproduce (5) for general $n$, and that spinons of an $\mathrm{SU}(n)$ chain transform under representation $\overline{\mathbf{n}}$ of $\mathrm{SU}(n)$ [25, 27]. It is also rather easy to see that for $\mathrm{SU}(2)$, there is a one-to-one correspondence between the eigenstates predicted by our formalism and the known asymptotic Bethe ansatz (ABA) solution [10, 12] in terms of motifs. It is further clear that the state counting [12, 15, 27], i.e., the requirement that the total dimension of the Hilbert space spanned by the many-spinon states must be $n^{N}$ for a system consisting of $N \mathrm{SU}(n)$ spins, works out automatically in the formalism, since the decomposition in representations of total spin given by the Young tableaux is complete and unaffected by our modification of the tableaux.

To establish the correctness of our proposal, we have compared the spectrum (classified in terms of total spin and momentum quantum numbers) of the $\mathrm{SU}(2)$ and the $\mathrm{SU}(3) \mathrm{HSM}$ up to 12 sites obtained numerically by diagonalizing (2) with the predictions of the tableau formalism, and find them identical. Finally, we have succeeded recently in showing that the predictions of the formalism agree with those made by the $\mathrm{ABA}$ for general $n$, as we will elaborate elsewhere [28]. This is still short of a rigorous proof as the applicability of the ABA to the model is heuristic as well, but in light of the success of the ABA solutions and the numerical work we carried out, we are confident that our formalism is correct.

Remarks: (a) For the polarized two-spinon states, there is a correspondence between the fermion occupations in the basis states (4) before projection and the tableau representations (see e.g. the $S_{\text {tot }}=1$ tableaux in Fig. 2). We conjecture that such a connection exists in general. If so, it would be desirable to have general rules for the construction of the required total spin representations with 


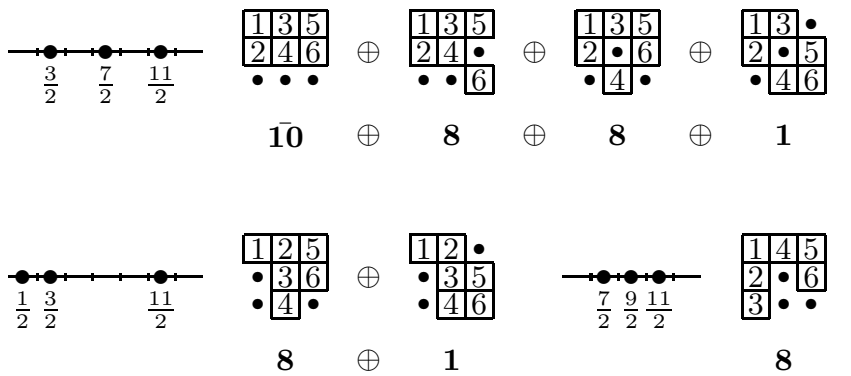

FIG. 4: Examples of allowed SU(3) spin representations for given SMNs in a chain with $N=6$ sites.

the fermions annihilated before projection such that the basis states with the same single-spinon momenta corresponding to different tableaux are orthogonal.

(b) The tableau formalism implies that the momenta of the spinons, as compared to the momenta of the fermions before projection, are shifted by $\frac{2 \pi}{N} \frac{1}{2 n}$ towards each other if they are in different columns of the tableau, and by the same amount away from each other if they are in the same column. (Since the "bare" momenta of the fermions are identical in the latter case, it is not possible to shift them towards each other.) The statistics of the spinons is hence that of fermions shifted by a statistical angle $\Delta \theta=\pi / n$ [18]. This implies $\theta=\pi\left(1-\frac{1}{n}\right)$ [17, 25] for spin-polarized spinons.

(c) The formalism can be used to calculate thermodynamic quantities. In this regard, it provides an alter- native to the "freezing trick" method of Sutherland and Shastry 29].

(d) The formalism provides, as a byproduct, the general rules which representations are possible for a given set of spinons with given single-particle momenta (see Fig. 4 for examples). These rules may also be interpreted in the framework of Yangian representation theory [30].

(e) Since the low energy physics of the HSM is described by the $\mathrm{SU}(n)$ level $k=1$ Wess-Zumino-Witten model [31], the rules for combining representations of spinons one may deduce form our formalism will apply to the quasiparticles of this theory as well. This connection has been exploited by Bouwknegt and Schoutens [17, 27], who obtained a significant body of results for $\mathrm{SU}(n)$ spin chains from conformal field theories.

In conclusion, we have established a one-to-one correspondence between the Young tableaux classifying the total spin representations of $N$ spins and the exact eigenstates of the the Haldane-Shastry model for a chain with $N$ sites. This correspondence allows us to label the manyspinon eigenstates in terms of their single-spinon momenta, which are spaced according to highly non-trivial rules. Since the spinons in the HSM are free in the sense that they only interact through their fractional statistics, the tableau formalism introduced here provides a general construction principle of the free spinon Hilbert space, the analog of a Fock space representation for spinons.

One of us (DS) was supported by the German Research Foundation (DFG) through GK 284.
[1] V. E. Korepin, N. M. Bogoliubov, and A. G. Izergin, Quantum Inverse Scattering Method and Correlation Functions (Cambridge University Press, Cambridge, 1997).

[2] N. Andrei, in Low-dimensional quantum field theories for condensed matter physicists, edited by S. Lundquist, G. Morandi, and Y. Lu (World Scientific, Singapore, 1992).

[3] L. D. Faddeev and L. A. Takhtajan, Phys. Lett. A 85, 375 (1981).

[4] H. Bethe, Z. Phys. 71, 205 (1931).

[5] R. B. Laughlin, Phys. Rev. Lett. 50, 1395 (1983).

[6] Quantum Hall Effect, edited by M. Stone (World Scientific, Singapur, 1992).

[7] F. D. M. Haldane, Phys. Rev. Lett. 60, 635 (1988).

[8] B. S. Shastry, Phys. Rev. Lett. 60, 639 (1988).

[9] F. D. M. Haldane, Phys. Rev. Lett. 66, 1529 (1991).

[10] N. Kawakami, Phys. Rev. B 46, 1005 (1992).

[11] F. D. M. Haldane, Z. N. C. Ha, J. C. Talstra, D. Bernard, and V. Pasquier, Phys. Rev. Lett. 69, 2021 (1992).

[12] Z. N. C. Ha and F. D. M. Haldane, Phys. Rev. B 47, 12459 (1993).

[13] F. H. L. Eßler, Phys. Rev. B 51, 13357 (1995).

[14] M. Greiter and D. Schuricht, Phys. Rev. B 71, 224424 (2005).

[15] F. D. M. Haldane, Phys. Rev. Lett. 67, 937 (1991).

[16] K. Schoutens, Phys. Rev. Lett. 79, 2608 (1997).

[17] P. Bouwknegt and K. Schoutens, Nucl. Phys. B 547, 501 (1999).
[18] M. Greiter, submitted to Nat. Phys.

[19] T. Yamamoto, Y. Saiga, M. Arikawa, and Y. Kuramoto, Phys. Rev. Lett. 84, 1308 (2000); J. Phys. Soc. Jpn. 69, 900 (2000).

[20] B. A. Bernevig, D. Giuliano, and R. B. Laughlin, Phys. Rev. B 64, 24425 (2001).

[21] S. Peysson, J. Phys. A: Math. Gen. 36, 7233 (2003).

[22] J. C. Talstra and F. D. M. Haldane, J. Phys. A: Math. Gen. 28, 2369 (1995).

[23] N. Kawakami, Phys. Rev. B 46, R3191 (1992).

[24] Z. N. C. Ha and F. D. M. Haldane, Phys. Rev. B 46, 9359 (1992).

[25] D. Schuricht and M. Greiter, Phys. Rev. B 73, 235105 (2006)

[26] see e.g. T. Inui, Y. Tanabe, and Y. Onodera, Group Theory and Its Applications in Physics (Springer, Berlin, 1996).

[27] P. Bouwknegt and K. Schoutens, Nucl. Phys. B 482, 345 (1996).

[28] M. Greiter and D. Schuricht, manuscript in preparation.

[29] B. Sutherland and B. S. Shastry, Phys. Rev. Lett. 71, 5 (1993).

[30] V. Chari and A. Pressley, L'Enseign. Math. 36, 267 (1990); A Guide to Quantum Groups (Cambridge University Press, Cambridge, 1998).

[31] P. Di Francesco, P. Mathieu, and D. Sénéchal, Conformal Field Theory (Springer, New York, 1997). 